\title{
NEDD8 Activating Enzyme
}

National Cancer Institute

\section{Source}

National Cancer Institute. NEDD8 Activating Enzyme. NCI Thesaurus. Code C95815.

NEDD8 activating enzyme is comprised of NEDD8-activating enzyme E1 regulatory subunit and NEDD8-activating enzyme E1 catalytic subunit. This protein complex modifies the C-terminus of NEDD8 to regulate protein neddylation, ubiquitination and catabolism. 